\title{
The Oscillator Model Improvement and Galloping Parameters Analysis of Conductors
}

\author{
Li Wei ${ }^{1, a}$, Lv Zhonghua ${ }^{1}$, Pan $\mathrm{Qi}^{1}$ \\ 1.State Grid Liaoning electric power company limited economic research institute,Liao Ning, Shenyang, 110000 \\ ateresa0517@sina.com
}

Key words: gallop, aerodynamic force, icing, 3d model

\begin{abstract}
The gallop of high voltage transmission line can lead a mass disaster to power facilities but it seems that people are not clear totally about the galloping mechanism. To make sure the safety of power grid, this paper studies deeply on the galloping oscillator model of transmission conductor and given the oscillator model which is globally expressed by dimensionless equation. In addition, it also pointed out that system damping cannot be ignored when a single degree of freedom oscillator model is adopted in analyzing the gallop of transmission lines. For the improved three degree of freedom oscillator model, the numerical solution of conductor galloping response is worked out by using the method of Runge Kutta to solve the differential equations. And the aerodynamic force of the conductors is developed into the third-order by Taylor's formula. The differential equations are simplified and solved by averaging and then the parameters will be analyzed.
\end{abstract}

\section{Introduction}

The gallop of transmission line is one kind of complex fluid - solid coupling vibration which is mainly decided by the conductor icing, wind-excitation, line structure and geographic condition. Based on the previous studies, this paper improved the traditional oscillator model and elaborated on it's derivation process to study it's forms of motion in the effect of wind-excitation. Combined with Den Hartog's galloping mechanism, this paper compares the changes of vertical displacement in different conditions to prove the rationality of derived models and do nonlinear vibration analysis to the conductor in different external condition to study the changes in conductors' forms of motion. Gain the critical wind velocity of conductors --- the galloping condition of conductors by Lyapunov stability theory, routh criterion and Horwitz criterion. According to the previous experimental result, change separately the icing-thickness and wind speed to do least square fitting about the aerodynamic coefficients and construct the equations of aerodynamic coefficient which has improved largely the correctness of parameter analysis. Then simulate the conductor galloping by adopting the assumed modes method. Firstly, the wave form of the conductor when it gallops is supposed as sinusoidal wave and then the motion of conductors are represented by three generalized coordinate. Analyze the stability of models by Horwitz criterion and gain the response of conductor galloping by solving equation set to know conductors' vibration and galloping in three directions. And then analyze it by changing the line structure and the environmental parameters.

\section{Improving the Oscillator Model}

\section{The traditional three degree of freedom oscillator model}

Taking the the position of the center of mass balance of the non-eccentric iced conductor when it is in in steady state as the origin of coordinates to construct a coordinate system like the following formula 1[1], 


$$
\left\{\begin{array}{l}
m \frac{d^{2} v}{d t^{2}}+c_{y} \frac{d v}{d t}+k_{y} v=F_{y} \\
m \frac{d^{2} \omega}{d t^{2}}+c_{z} \frac{d \omega}{d t}+k_{z} \omega=F_{z} \\
\mathrm{I} \frac{d^{2} \theta}{d t^{2}}+c_{\theta} \frac{d \theta}{d t}+\kappa_{\theta} \theta=F_{M}
\end{array}\right.
$$

$\mathrm{t}$ represents time; $\mathrm{v}$ represents the displacement in the direction of $\mathrm{y}$ (vertical); $\mathrm{w}$ represents the displacement in the direction of $\mathrm{z}$ (horizontal); $\theta_{\text {represents the torsional angle of conductors; } \mathrm{m}}$ represents the mass per unit length of conductors including the icing; I represents the rotary inertia of conductors including the icing.

\section{Improving the Galloping Model of Conductors}

The iced conductor mainly vibrates in across-wind, down wind and reverses course and the generalized coordinates of vibration modal in each direction can be used to represent the displacement in this three directions. We suppose that the vibration modal of each order in all direction are all sine curves. Thus we can represent it by multiplying the generalized displacement in some point like s and vibration modal, it is,

$$
\left\{\begin{array}{l}
v(s, t)=\sin \left(\frac{n \pi s}{L}\right) V(t) \\
\omega(s, t)=\sin \left(\frac{n \pi s}{L}\right) W(t) \\
\theta(s, t)=\sin \left(\frac{n \pi s}{L}\right) \Theta(t)
\end{array}\right.
$$

Among which , $\mathrm{V}(\mathrm{t}) 、 \mathrm{~W}(\mathrm{t})$ and $\theta(t)$ represents respectively the generalized displacements of certain point $s$ of conductors at time $t$ in across-wind, down wind and reverses course; $\mathrm{V}(\mathrm{s}, \mathrm{t}) 、 \mathrm{~W}(\mathrm{~s}, \mathrm{t})$ and $\theta(s, t)$ represents the displacements of certain point $s$ of conductors at time $t$ in across-wind, down wind and reverses course. $\sin \left(\frac{n \pi S}{L}\right)$ represents the modal of transmission conductor in n-th $\operatorname{order}^{[2]}$.

The inclination angle of the conductor section determines the magnitude of aerodynamic force of conductors on this section. When horizontal wind and the conductor are in vertical state, the inclination angles of the conductor mainly include the twist angle of itself $\theta$ and the inclination angle $\beta$. The inclination angle is formed by the horizontal wind speed and twist speed of conductors. The following is the free-body diagram of conductors when it is stressed by the inclination angle,
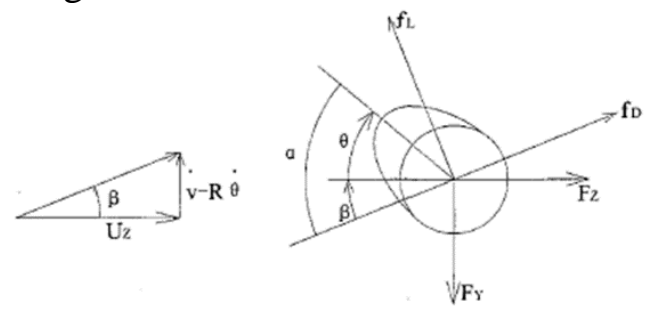

Figure 1 inclination angle

Among which, R represents the characteristic radius of conductors, Uz represents the horizontal wind speed. At the point $\mathrm{S}$, the total inclination angle of the conductor $\alpha$ is

$$
\alpha(s, t)=\theta(s, t)+\frac{1}{U_{Z}} \dot{v}(s, t)-\frac{R}{U_{Z}} \dot{\theta}(s, t)
$$

Substitute the formula (2) into the formula (3), we can get the expression form in generalized coordinate of the inclination angle. 


$$
\alpha(s, t)=\sin \left(\frac{n \pi S}{L}\right) \Theta(t)+\frac{1}{U_{\text {rel }}} \sin \left(\frac{n \pi S}{L}\right) \dot{V}(t)-\frac{R}{U_{\text {rel }}} \sin \left(\frac{n \pi S}{L}\right) \dot{\Theta}(s, t)
$$

Because in $U_{\mathrm{rel}}=\frac{U_{Z}-\dot{\omega}}{\cos \beta}$, Uz is much larger than $W$, and $\beta$ and $\arctan =\frac{R \dot{\theta}}{U_{Z}-\dot{\omega}}$ can be ignored for they are little. So $U_{Z} \approx U_{\text {rel }}$.

The aerodynamic force taken in per unit length of the iced conductors can be decomposed as the buoyancy $f_{D}$ which is along the relative wind direction, the buoyancy $f_{L}$ which is vertical with the relative wind direction and the torsion $f_{M}$, and their expression forms are relatively,

$$
\left\{\begin{array}{l}
f_{D}=\frac{1}{2} \rho U_{r e I}^{2} d C_{D} \\
f_{L}=\frac{1}{2} \rho U_{r e I}^{2} d C_{L} \\
f_{M}=\frac{1}{2} \rho U_{r e I}^{2} d C_{M}
\end{array}\right.
$$

We suppose that the displacements of a certain point on the conductor are respectively $\mathrm{v}(\mathrm{s}, \mathrm{t}), \mathrm{w}(\mathrm{s}, \mathrm{t})$ and $\theta(s, t)$, so the works done by the conductors' aerodynamic force in this point and the corresponding displacement are equal to the works done by the corresponding generalized force $Q_{D}$, $Q_{L}$ and $Q_{M}$ of aerodynamic force in corresponding displacements, it is,

$$
Q_{i}=\int_{0}^{L} \sin \left(\frac{n \pi S}{L}\right) f_{i} d S
$$

Among which, $Q_{D}, Q_{L}$ and $Q_{M}$ are represented by $Q_{i}, f_{i}$ represents respectively the buoyancy $f_{L}$, the resistance $f_{D}$ and the torsion $f_{M} \cdot Q_{i}$ represents the conductors' aerodynamic force which is along the relative wind direction. If it is converted as the directions of Y-axis and X-axis in a fixed coordinate, we can gain this, ${ }^{[4]}$

$$
\left\{\begin{array}{c}
F_{Y}=-Q_{L} \cos \beta-Q_{D} \sin \beta \\
F_{Z}=-Q_{L} \sin \beta+Q_{L} \cos \beta \\
F_{M}=Q_{M}
\end{array}\right.
$$

Among which, $F_{Y}, F_{Z}$ and $F_{M}$ represents relatively the generalized aerodynamic force in this three directions.

\section{The Analysis of Conductor Galloping Parameters}

The phase plane portraits of vertical motion:

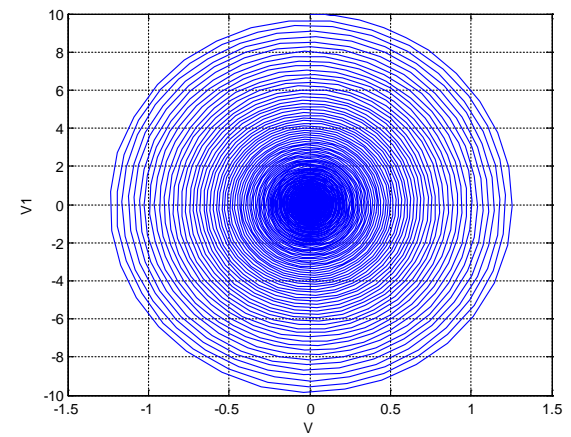

a) $U_{r e l}$ is $5 \mathrm{~m} / \mathrm{s}$

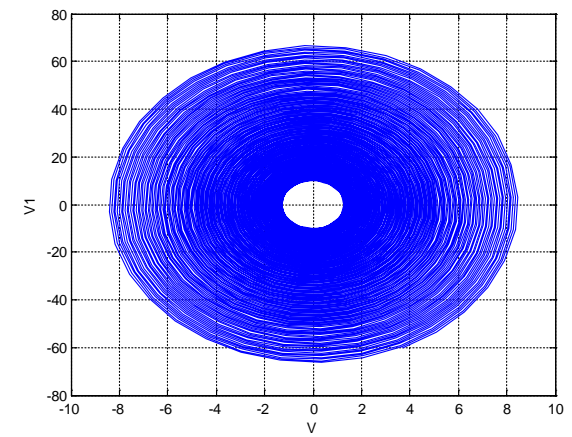

b) $U_{r e l}$ is $10 \mathrm{~m} / \mathrm{s}$ 


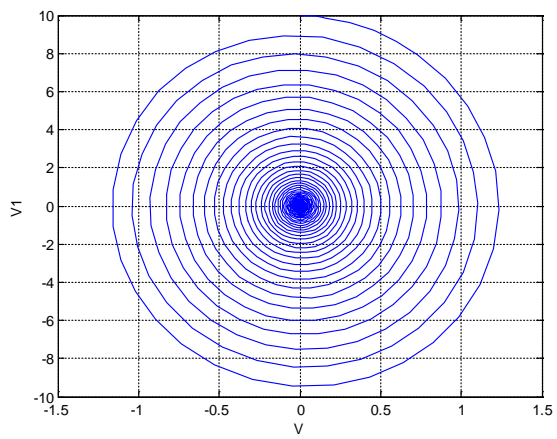

c) $U_{r e l}$ is $20 \mathrm{~m} / \mathrm{s}$

Figure 2 The phase plane portraits of vertical motion

It is observed that the conductor did not gallop and the center of it is very concentrate when the wind speed is $5 \mathrm{~m} / \mathrm{s}$ which shows that the vertical displacements of conductors are mainly minute displacements at this moment. The speed in vertical motion of conductors are all small and the diagram is very close to a round shape. The conductors' displacement is similar with the change amplitude of the speed of the motion.

With the increase of the wind speed, the conductor's largest vertical displacement increased to $8 \mathrm{~m}$ from $1.3 \mathrm{~m}$ and decreased to $1.3 \mathrm{~m}$ finally. And the largest vertical velocity increased to $60 \mathrm{~m} / \mathrm{s}$ from $10 \mathrm{~m} / \mathrm{s}$ and decreased to $10 \mathrm{~m} / \mathrm{s}$ finally. That is the conductor firstly mainly do small amplitude vibration and then turn to large amplitude vibration and finally turn back to do small amplitude vibration. In a similar way, the changes of the minimum value of displacements and velocity are similar.

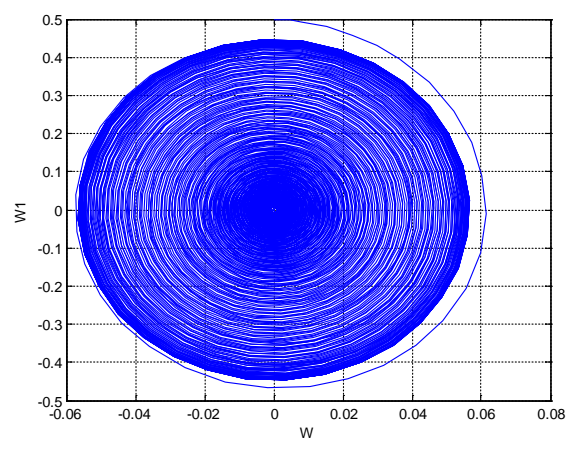

a) $U_{r e l}$ is $5 \mathrm{~m} / \mathrm{s}$

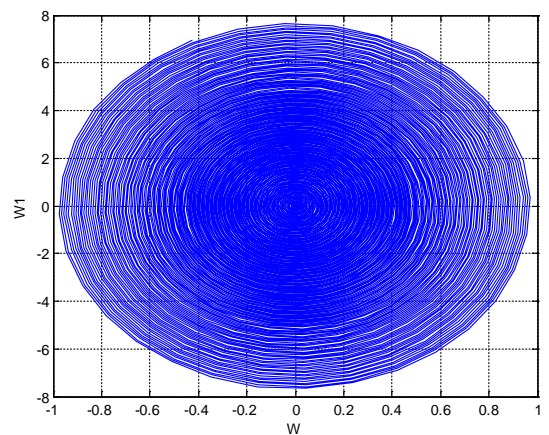

b) $U_{r e l}$ is $10 \mathrm{~m} / \mathrm{s}$

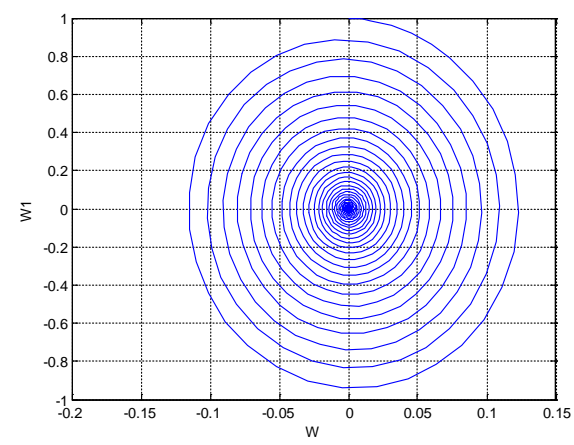

c) $U_{r e l}$ is $20 \mathrm{~m} / \mathrm{s}$

Figure 3 The phase plane portraits of vertical motion

It is observed that when the wind sped is $5 \mathrm{~m} / \mathrm{s}$, the conductors did not gallop and are very concentrate in each section. The diagram is close to a round shape which shows that at this wind speed, the changing degrees of transverse displacements and the velocity are very close. When the wind speed is $10 \mathrm{~m} / \mathrm{s}$, the conductors galloped and are very concentrated in each section. The diagram takes a shape of an oval with a rather large horizontal axis which shows that at this wind speed, the 
changing degree of the displacements is bigger than that of conductors' velocity. The displacement and the velocity values both increased. When the wind speed is $20 \mathrm{~m} / \mathrm{s}$, the conductors' vibration mainly concentrates in small numerical area and the displacements and velocity values both decreased which is generally similar with those when the wind speed is $5 \mathrm{~m} / \mathrm{s}$.

With the increase of the wind speed, the largest transverse displacement of conductors increased to $1 \mathrm{~m}$ from $0.06 \mathrm{~m}$ and decreased to $0.12 \mathrm{~m}$ finally. And the maximum value of vertical velocity increased to $8 \mathrm{~m} / \mathrm{s}$ from $0.5 \mathrm{~m} / \mathrm{s}$ and decreased to $1 \mathrm{~m} / \mathrm{s}$ finally. In a similar way, the minimum value of the displacement and velocity are similar. While the transverse section of conductors firstly do small amplitude vibration and then turn to large amplitude vibration and finally turn back to do small amplitude vibration.

\section{Conclusion}

For under the traditional oscillator, the conductor galloping is the form of simple harmonic motion, this paper substitutes the steady-state horizontal wind with the relative wind speed to make the results close to the reality. The nondimensionalization of equation can highlight the influences of damping parameter, line structure parameters and meteorological parameter on conductor galloping and thus do parameter analysis about it. When the conductor did not gallop, the displacements in three directions decrease with the increase of time and finally tend to a certain minimum value and then do small amplitude vibration around it. When the conductors gallop, the displacements in three directions increase with the increase of time and tend to a certain maximum value and then decrease with the increase of time and tend to a certain minimum value and stop increasing. This mainly because the conductors' vibration are one kind of non-linear vibration and even if it loses the stability, it can still be steady at a certain limited value. And only the linear system will keep increasing when it lose the stability.

\section{Reference}

[1] Yishuang Geng, Jin Chen, Ruijun Fu, Guanqun Bao, Kaveh Pahlavan, Enlighten wearable physiological monitoring systems: On-body rf characteristics based human motion classification using a support vector machine, IEEE transactions on mobile computing, 1(1), 1-15, Apr. 2015

[2] Shuang Zhou, Liang Mi, Hao Chen, Yishuang Geng, Building detection in Digital surface model, 2013 IEEE International Conference on Imaging Systems and Techniques (IST), Oct. 2012

[3] Zhihan Lv, Alex Tek, Franck Da Silva, Charly Empereur-Mot, Matthieu Chavent, and Marc Baaden. "Game on, science-how video game technology may help biologists tackle visualization challenges." PloS one 8, no. 3 (2013): e57990.

[4] Yu Song, et al.. A Rapid and High Reliable Identify Program for Nighttime Pedestrians. Infrared Physics \& Technology. 2015.

[5] Jie He, Yishuang Geng, Fei Liu, Cheng Xu, CC-KF: Enhanced TOA Performance in Multipath and NLOS Indoor Extreme Environment, IEEE Sensor Journal, 14(11), 3766-3774, Nov. 2014 